\title{
Anti-N-methyl-D-aspartate receptor encephalitis A case report
}

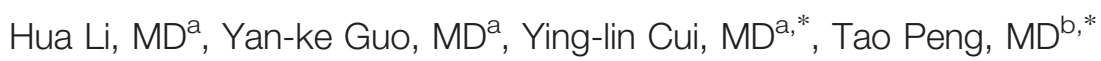

\begin{abstract}
Rationale: Anti-N-methyl-D-aspartate receptor (anti-NMDAR) encephalitis is an auto-immune and paraneoplastic encephalitis with prominent neuropsychiatric manifestations. The $\mathrm{N}$-methyl-D-aspartate receptor is located in the forebrain and hippocampus and plays a role in learning and memory.

Patient concerns: A 29-year-old female patient with anti-NMDAR encephalitis, was reported and we also reviewed the literature and summarised the characteristics of the cases.

Diagnoses: In the present study, we reported 1 patient with anti-NMDAR encephalitis diagnosed by the detection of anti-NMDAR antibodies in serum and cerebrospinal fluid (CSF).

Interventions: The patient received glucocorticoids and anti-epilepsy treatment as well as human immunoglobulin treatment.

Outcomes: After treatment, the patient gradually regained consciousness and was discharged after 3 months of rehabilitation. At the follow-up 2 months later, the patient had the sequelae of memory impairment and limb movement disorders.

Lessons: An accurate early diagnosis and active treatment are crucial to the improvement in the prognosis of patients with antiNMDAR receptor encephalitis.

Abbreviations: $A D A=$ adenosine deaminase, anti-NMDAR $=$ anti-N-methyl-D-aspartate receptor, CSF $=$ cerebrospinal fluid, FLAIR = fluid-attenuated inversion recovery, Glu = glucose, $\mathrm{LDH}=$ lactate dehydrogenase, $\mathrm{MRI}=$ magnetic resonance imaging, $\mathrm{T} 2 \mathrm{WI}=\mathrm{T} 2$ weighted images.
\end{abstract}

Keywords: anti-N-methyl-D-aspartate receptor, encephalitis

\section{Introduction}

Anti-N-methyl-D-aspartate receptor (anti-NMDAR) encephalitis is a novel type of autoimmune encephalitis that is mediated by anti-NMDAR antibodies and mainly involves the limbic system. The N-methyl-D-aspartate receptor is located in the forebrain and hippocampus and plays a role in learning and memory. In this disease, the NR1 subunit of the NMDA receptors is targeted by antibodies resulting in internalization of NMDAR and progressive decline of NMDAR-associated synaptic functions resulting in neurological and psychiatric manifestations. It often occurs in young women, with a male to female ratio of approximately $1: 3 .^{[1,2]}$ The average age of onset of symptoms is 21 years, although patients have been described in patients ranging from 8 months to 85 years. ${ }^{[2,3]}$ The clinical manifes-

\footnotetext{
Editor: N/A.

The authors have no conflicts of interest to disclose.

a Department of Emergency, Henan Traditional Chinese Medicine Hospital, Zhengzhou, Henan, ${ }^{b}$ Department of Nephrology, Shandong University Qilu Hospital, Jinan, Shandong, China.

* Correspondence: Tao Peng, Ying-lin Cui, Department of Nephrology, Shandong University Qilu Hospital, Jinan, Shandong 250012, China

(e-mail: sesame_oil@126.com).

Copyright (C) 2018 the Author(s). Published by Wolters Kluwer Health, Inc.

This is an open access article distributed under the terms of the Creative Commons Attribution-Non Commercial License 4.0 (CCBY-NC), where it is permissible to download, share, remix, transform, and buildup the work provided it is properly cited. The work cannot be used commercially without permission from the journal.

Medicine (2018) 97:50(e13625)

Received: 17 July 2018 / Accepted: 19 November 2018

http://dx.doi.org/10.1097/MD.0000000000013625
}

tations are complex, and a missed diagnosis or misdiagnosis can easily occur. Early diagnosis and timely immunotherapy have important significance for this disease. Anti-NMDAR encephalitis was first described in 1997 in 2 isolated patient reports of young females with ovarian teratomas presenting with psychiatric manifestations. ${ }^{[4]}$ In the present study, we reported 1 patient with anti-NMDAR encephalitis diagnosed by the detection of anti-NMDAR antibodies in serum and cerebrospinal fluid (CSF) and discusses the literature in order to increase clinicians' understanding of anti-NMDAR encephalitis.

\section{Case report}

A 29-year-old female patient was admitted to the hospital with dizziness and headache for 40 days; aggravation of symptoms together with consciousness disturbance for 1 month. Forty days before admission, without obvious causes, the patient had dizziness and a headache as well as nausea, vomiting, and a sensation of rotation with the eyes open, which were alleviated after the eyes were closed. The headache mainly consisted of persistent pain in the bilateral temporal lobes, and the degree of pain was unbearable. The patient had a fever, with the highest temperature reaching $38.9^{\circ} \mathrm{C}$. With the increased body temperature, the patient had an aggravated headache, body fatigue, and muscle aches, but showed no cough, sputum, night sweats, chills, or other symptoms. The patient had been treated at a local clinic. A lumbar puncture examination of the CSF showed a pressure of $320 \mathrm{~mm} \mathrm{H}_{2} \mathrm{O}$. No abnormality was found in the cranial magnetic resonance imaging (MRI). Treatment for viral encephalitis led to poor treatment efficacy. The patient's condition continued to deteriorate, with loss of consciousness, intermittent convulsions, and weak spontaneous breathing. Tracheal intubation was 
conducted for mechanical ventilation-assisted breathing, and a tracheotomy was performed later. The patient was then transferred to our hospital. Examination upon admission revealed that the patient had a body temperature of $36.8^{\circ} \mathrm{C}$, showed confusion of consciousness, and did not cooperate in the examination of advanced intelligence activities. Bilateral pupils were equal and round, approximately $2.0 \mathrm{~mm}$ in diameter, and the patient showed sensitive light reflection. The patient did not cooperate with the limb muscle force examination and had reduced muscle tension, positive tendon reflexes $(+)$, and a bilateral negative Babinski sign $(-)$. The CSF examination revealed a pressure of $140 \mathrm{mmH}_{2} \mathrm{O}$, glucose (Glu) $6.25 \mathrm{mmol} / \mathrm{L}$, lactate dehydrogenase (LDH) $129 \mathrm{u} / \mathrm{L}, \mathrm{Cl}^{-} 126 \mathrm{mmol} / \mathrm{L}$, protein quantitation $487 \mathrm{mg} / \mathrm{L}$, adenosine deaminase (ADA) $4.2 \mathrm{u} / \mathrm{L}$, nucleated cell count $5.0 \times 10^{6} / \mathrm{L}$, and a ratio of mononuclear to multinuclear cells of 4:6. The CSF was negative for the virus antibody. During re-examination, cranial MRI findings revealed abnormally high signals on the right hippocampus, insula, and medial temporal lobe in T2 weighted images (T2WI) and fluidattenuated inversion recovery (FLAIR) images (Figs. 1 and 2). Serum and CSF were positive for antibodies against NMDAR. The initial diagnosis of anti-NMDAR receptor encephalitis was made, and the patient received glucocorticoids and anti-epilepsy treatment as well as human immunoglobulin treatment $(15 \mathrm{~g} / \mathrm{d}$ for 5 days, followed by once per week for 3 weeks, and then once per month for 2 months). No tumor was revealed on a chest and abdomen computed tomography (CT) scan. After treatment, the patient gradually regained consciousness and was discharged after 3 months of rehabilitation. At the follow-up 2 months later, the patient had the sequelae of memory impairment and limb movement disorders.

The patient and his family agreed with the authors of this manuscript to freely apply his personal and medical information, including published articles and further scientific research, and the patient already signed the informed consent.

\section{Discussion}

Anti-NMDAR encephalitis was first reported by Cai X. et al ${ }^{[5]}$ in 2005 and was named for the first time in 2007. ${ }^{[6]}$ The clinical manifestations are characterized by mental disorders, disturbances

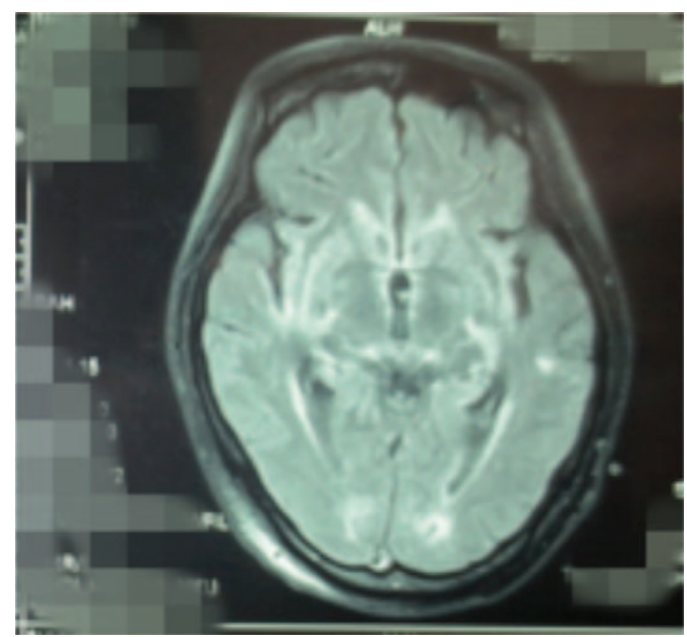

Figure 1. MRI scan FLAIR image: high signal of right hippocampus and bilateral island patch. FLAIR=fluid-attenuated inversion recovery, $M R I=$ magnetic resonance imaging.

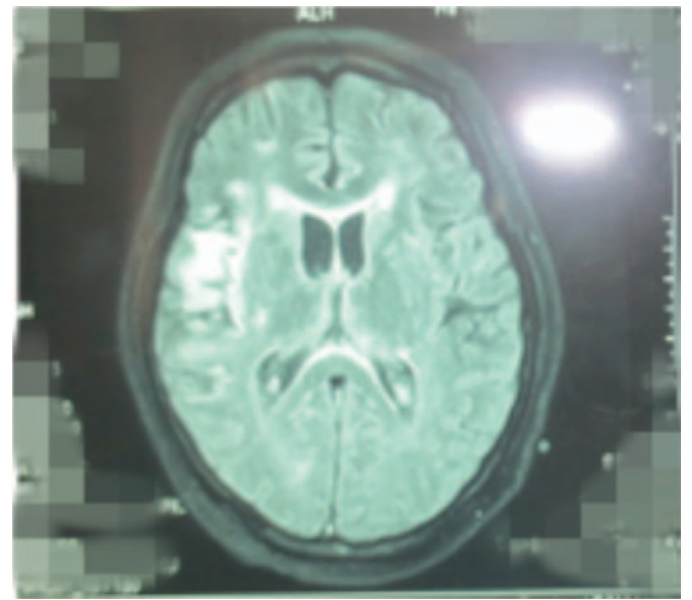

Figure 2. MRI scan FLAIR image: high signal on the right side of the temporal lobe. FLAIR = fluid-attenuated inversion recovery, MRI= magnetic resonance imaging.

of consciousness, epilepsy, mouth-face-tongue involuntary movements, insufficiency of ventilation, and autonomic dysfunction, with or without associated tumors, and with teratomas as the most common associated tumors. It is particularly common in young female patients with ovarian teratomas. No tumors were found during the clinical diagnosis and treatment of the patient. The patient had the symptom of fever in the early stage, and developed hypoventilation syndrome. The patient gradually showed disturbances of consciousness, cognitive decline, and epileptic symptoms. This is consistent with the clinical manifestations of previously reported cases. ${ }^{[7]}$ Viaccoz et al reported that the majority of anti-NMDAR encephalitis patients with seizures and mouth-face-tongue involuntary movements were male and that partial seizures were more common. ${ }^{[8-10]}$ In the present study, the patient had epilepsy and typical mouth-face-tongue involuntary movements and was diagnosed as having anti-NMDAR encephalitis after the positive detection of anti-NMDAR antibodies. Therefore, this suggests that for male patients suffering from mental disorders, epileptic seizures, and involuntary mouth-facetongue movements, a serum or CSF anti-NMDAR antibody test should be conducted as soon as possible to confirm the diagnosis. At present, the incidence of anti-NMDAR encephalitis is not clear. However, with in-depth studies conducted in recent years, the number of clinical reports has gradually increased, which may be related to the continuous improvement of detection ability.

There is no uniform standard for the diagnosis of antiNMDAR encephalitis. Currently, diagnosis is determined based on the patient's serum and CSF anti-NMDAR antibodies, characteristic clinical manifestations, head MRI changes, and an effective response to immunotherapy. Among these diagnostic tests, positive serum and CSF anti-NMDAR antibodies have the highest specificity. ${ }^{[3]}$ In this paper, the patient was positive for serum and CSF anti-NMDAR antibodies and the symptoms were relieved after immunotherapy. Patients with anti-NMDAR encephalitis present no specific changes in the routine biochemical examination of CSF, and often show mononuclear/lymphocyte hyperplasia, mild or moderate protein elevation, usually an elevated IgG index, or the appearance of oligoclonal bands. The early cranial MRI findings of anti-NMDAR encephalitis also present no specificity. As the disease progresses, T2WI and diffusion-weighted imaging (DWI) can show abnormal signals in 
the medial temporal lobes, and abnormally high signals in the cerebellum, cortex, basal ganglia, and brainstem, indicating demyelinating changes, and usually transient lesions or mild meningeal enhancement. The Patient showed abnormally high signals in T2WI and FLAIR images in the bilateral hippocampus, insula, and medial temporal lobes. The MRI results of the patient were basically consistent with those reported in the literature. ${ }^{[1]}$

Due to the frequent acute onset of anti-NMDAR encephalitis, and lacking specific features in imaging examinations, antiNMDAR encephalitis is often confused with viral encephalitis in the early stages. ${ }^{[12]}$ Viral encephalitis also manifests itself as an acute-onset, rapidly progressing disease, and has symptoms such as fever, headache, vomiting, psychiatric symptoms, epilepsy, and disturbance of consciousness during the course of the disease. For patients with involuntary mouth-face-tongue movements, central hypoventilation syndrome, and a poor response to antiviral therapy during disease diagnosis and treatment, anti-NMDAR encephalitis should be considered. Serum and CSF-specific pathogens should be detected promptly, cranial MRI should be examined again, and patients should be given sufficient glucocorticoid and immunoglobulin treatment, and if necessary, treated with immunosuppressive drugs such as cyclophosphamide. ${ }^{[13]}$ In this paper, the patient was treated based on the diagnosis of viral encephalitis at an early stage, but the effect of the antiviral therapy was poor. Serum and CSF anti-NMDAR antibody tests were performed, which led to positive results, and the diagnosis of anti-NMDAR encephalitis was finally confirmed. Typical mouth-face-tongue involuntary movements occurred at the early onset of the disease. The diagnosis and treatment were timely, and the prognosis was better.

Early large doses of glucocorticoids and gamma globulin are often used as first-line treatment drugs for anti-NMDAR encephalitis and are effective in most patients. ${ }^{[14]}$ If the firstline treatment is not effective, the second-line treatment (cyclophosphamide or rituximab) is often effective. ${ }^{[15]}$ In the present study, the patient was treated with glucocorticoids combined with gamma globulin after diagnosis, and also received symptomatic treatment. After treatment, patient was clinically cured upon discharge, improved symptoms after discharge for 1 month, gradually regained ventilation function, and had minor sequelae of movement and cognitive dysfunction.

In summary, for patients with acute or subacute onset, psychomotor abnormalities, and epileptic seizures, and especially with the characteristic clinical manifestations of mouth-facetongue and limb involuntary movements and central ventilatory dysfunction, it is necessary to consider the possibility of antiNMDAR encephalitis. Serum and CSF anti-NMDAR antibody detection should be conducted to confirm the diagnosis, thus achieving early treatment and improving the prognosis.

\section{Author contributions}

Conceptualization: Hua Li.

Project administration: Tao Peng.

Writing - original draft: Yan-ke Guo.

Writing - review \& editing: Ying-lin Cui.

\section{References}

[1] Gable MS, Sheriff H, Dalmau J, et al. The frequency of autoimmune Nmethyl-D-aspartate receptor encephalitis surpasses that of individual viral etiologies in young individuals enrolled in the California Encephalitis Project. Clin Infect Dis 2012;54:899-904.

[2] Liu CY, Zhu J, Zheng XY, et al. Anti-N-Methyl-D-aspartate receptor encephalitis: a severe, potentially reversible autoimmune encephalitis. Mediators Inflamm 2017;2017:6361479.

[3] Titulaer MJ, McCracken L, Gabilondo I, et al. Treatment and prognostic factors for long-term outcome in patients with anti-NMDA receptor encephalitis: an observational cohort study. Lancet Neurol 2013;12:157-65.

[4] Gumbinger C, Hametner C, Wildemann B, et al. Administration of isoflurane-controlled dyskinetic movements caused by anti-NMDAR encephalitis. Neurology 2013;80:1997-8.

[5] Cai X, Zhou H, Xie Y, et al. Anti-N-methyl-D-aspartate receptor encephalitis associated with acute Toxoplasma gondii infection: a case report. Medicine (Baltimore) 2018;97:e9924.

[6] Dalmau J, Tüzün E, Wu HY, et al. Paraneoplastic anti-N-methl-Daspartate receptor encephalitis associated with ovarian teratoma. Ann Neurol 2007;61:25-36.

[7] Dalmau J, Lancaster E, Marinez-ernan-dezE. Clinical experience and laboratory investigations in patients with anti-NMDA Rencephalitis. Lancet Neurol 2011;10:63-74.

[8] Li L, Sun L, Du R, et al. Application of the 2016 diagnostic approach for autoimmune encephalitis from Lancet Neurology to Chinese patients. BMC Neurol 2017;17:195.

[9] Viaccoz A, Desestret V, Ducray F, et al. Clinical specificities of adult male patients with NMDA receptor antibodies encephalitis. Neurology 2014;82:556-63.

[10] Lee KW, Liou LM, Wu MN. Fulminant course in a patient with anti-Nmethyl-D-aspartate receptor encephalitis with bilateral ovarian teratomas: a case report and literature review. Medicine (Baltimore) 2018;97: e0339.

[11] Nazif TM, Vázquez J, Honig LS, et al. Anti-N-methyl-D-aspartate receptor encephalitis: an emerging cause of centrally mediated sinus node dysfunction. Europace 2012;14:1188-94.

[12] Wang X, Lai Y, Li P, et al. Massive idiopathic spontaneous hemothorax complicating anti-N-methyl-d-aspartate receptor encephalitis: a case report. Medicine (Baltimore) 2018;97:e13188.

[13] Gomes Ferreira M, Lapresa Alcalde V, García Sánchez MH, et al. Successful treatment of anti-NMDA receptor encephalitis with early teratoma removal and plasmapheresis: a case report. Medicine (Baltimore) 2018;97:e11325.

[14] Lebas A, Husson B, Didelot A, et al. Expanding spectrum of encephalitis with NMDA receptor antibodies in young children. Child Neural 2010;25:742-5.

[15] Liu X, Yan B, Wang R, et al. Seizure outcomes in patients with antiNMDAR encephalitis: a follow-up study. Epilepsia 2017;58:2104-11. 